\title{
Ambulante Palliativversorgung in Westfalen-Lippe
}

\author{
Eine Follow-up-Erhebung fünf Jahre nach Einführung \\ einer strukturierten Versorgung im häuslichen Umfeld
}

\author{
E. A. Lux ${ }^{1}$, U. Hofmeister ${ }^{2}$, R. Bornemann ${ }^{3}$
}

\section{Zusammenfassung}

Hintergrund und Fragestellung I Seit 2009 kooperieren in Westfalen-Lippe Hausärzte mit Palliativärzten, die in regionalen Palliativmedizinischen Konsiliardiensten (PKD), organisiert sind und von Koordinatoren unterstützt werden. Vertragliche Grundlage ist die „Vereinbarung zur Umsetzung der ambulanten palliativmedizinischen Versorgung von unheilbar erkrankten Patienten im häuslichen Umfeld“.

Methodik | Seit 2009 erfolgt eine strukturierte Dokumentation dieser Tätigkeit. Wir werteten die Daten für die Jahre 2009/10 bis 2014 nach Region, Anzahl der betreuten Patienten und Sterbeort aus.

Ergebnisse I In Westfalen-Lippe starben 2014 etwa 91000 Menschen, von denen etwa 19,4\% (17699 Patienten) ein umfassendes Angebot ambulanter palliativmedizinischer Versorgung erhielten. Dieser Anteil lag 2009/2010 mit 5682 Palliativpatienten noch bei $6 \%$. Die Anzahl der Patienten, die zu Hause starben, stieg zwischen
2009/2010 und 2014 von ca. $70 \%$ auf ca. $75 \%$ an, wohingegen im Krankenhaus (Normalstation) 2014 nur noch ca. 9\% der Palliativpatienten starben, gegenüber 13\% 2009/2010. Dieser Trend zeigte sich sowohl in städtischen als auch in ländlichen Regionen.

Folgerung I Die Anzahl der Patienten, welche in Westfalen-Lippe bei niederschwelligem Zugang zu den ambulanten palliativen Versorgungsstrukturen durch hausärztlich orientierte PKD versorgt werden, hat sich während der letzten fünf Jahre etwa verdreifacht. Die Zusammenarbeit von Hausärzten und PKD ermöglicht einen unbürokratischen Übergang von der allgemeinen zur spezialisierten ambulanten Palliativversorgung. Wesentlicher Garant des multidisziplinären und multiprofessionellen Arbeitens sind die in den PKD tätigen, in Palliativversorgung ausgebildeten und erfahrenen Koordinatoren. Die regional unterschiedliche Qualität und Quantität der Versorgung soll durch regelmäßige und strukturierte Datenerfassung und -analyse in Sinne der Patienten verbessert werden.

\section{Einleitung}

Seit dem 01.04.2009 erfolgt im Bereich der Kassenärztlichen Vereinigung Westfalen-Lippe (KVWL) die ambulante palliativmedizinische Versorgung auf der Basis der „Vereinbarung zur Umsetzung der ambulanten palliativmedizinischen Versorgung von unheilbar erkrankten Patienten im häuslichen Umfeld“ nach $\S 116$ b SGB V [9]. Diese „Vereinbarung“ ermöglicht, im Gegensatz zu anderen Verträgen der spezialisierten ambulanten Palliativversorgung (SAPV), eine frühe Integration von Palliativmedizin und -pflege.

Das Tätigwerden ist dabei nicht explizit an das Vorliegen einer ausgeprägten und komplexen Symptomatik mit besonderem Betreuungsaufwand geknüpft. Als Kriterium für die Aufnahme eines Patienten genügt vielmehr, dass eine in Wochen bis Monaten zum Tode führende Erkrankung vorliegt. Dadurch wird die Rolle der Hausärzte im Rahmen der Palliativversorgung gestärkt.
Die Richtlinie des Gemeinsamen Bundesausschusses zur Verordnung von spezialisierter ambulanter Palliativversorgung und das Sozialgesetzbuch V ( $§ 132 \mathrm{~d}$ Abs. 2) regeln den Rechtsanspruch auf SAPV. Bei ausgeprägter Symptomatik kann die hausärztliche Versorgung in Kooperation mit Palliativärzten und Koordinatoren in palliativ-medizinischen Konsiliardiensten (PKD) unter Einbindung von spezialisierten Palliativpflegediensten (nach § 132a Abs. 2 SGB V) erbracht werden. Konkret besteht die Möglichkeit, qualifizierte Palliativärzte als Konsiliarii oder Behandler rund um die Uhr zur Verfügung zu haben. Solche Übergänge von allgemeiner ambulanter Palliativversorgung (AAPV) zu SAPV sind unbürokratisch geregelt. Der Mehraufwand der AAPV wird inzwischen im Rahmen der „Vereinbarung" angemessen abgebildet: in Form einer Einschreibepauschale sowie der extrabudgetären Vergütung von Hausbesuchen.

Inzwischen nehmen in Westfalen-Lippe ca. 90\% der Hausärzte an diesem System Teil, unterstützt durch ca. 260 Palliativmediziner sowie ca.
Institute

1 Klinik für Schmerz- und Palliativmedizin, Klinikum St.-Marien-Hospital Lünen Fakultät für Gesundheit, Universität WittenHerdecke

2 Berufsverband der Palliativmediziner in Westfalen-Lippe e. V., Münster

3 Innere Klinik, Klinikum Bielefeld; Fakultät für Gesundheitswissenschaften, Universität Bielefeld

\section{Korrespondenz}

Dr. med. Eberhard Albert Lux Klinik für Schmerz- und Palliativmedizin Klinikum St.-Marien-Hospital Lünen

Altstadtstr. 23, 44534 Lünen Tel.: +49 (0)2306/77-2920 Fax: +49 (0)2306/77-2921 drlux@web.de 
100 Koordinatoren. Seit 2013 ist ein externer, nicht weisungsgebundener Beauftragter tätig, der unabhängig von der Kassenärztlichen Vereinigung oder den PKD zur Sicherung und Verbesserung der Versorgungsqualität beitragen soll. Der Wirkungsbereich der KVWL erstreckt sich über ein Gebiet in Nordrhein-Westfalen von ca. $21000 \mathrm{~km}^{2}$ mit ca. 8,3 Millionen Einwohnern, entsprechend einer Bevölkerungsdichte von ca. 395 Einwohnern $/ \mathrm{km}^{2}$. Pro Jahr sterben hier etwa 91000 Menschen [10].

PKD wurden 2009 gegründet und sind regional, auf Basis der administrativen Kreise, organisiert. Die Versorgungsstruktur in Westfalen-Lippe unterscheidet sich vom klassischen Arbeitskonzept regionaler SAPV-Teams. Daher bestand Erforschungsbedarf des neuen AAPV-Konzeptes: Ende 2010 wurden die einzelnen PKD erstmals zur Struktur- und Ergebnisqualität der von ihnen erbrachten ambulanten Palliativversorgung befragt $[12,13]$.

Seit 2012 erfolgt durch die PKD eine quartalsweise Meldung der erbrachten Leistungen an die KVWL:

- Anzahl Patienten in Voll- und Teilversorgung

- Betreuungsdauer

- Sterbeorte

Ziel der vorliegenden Studie war es, zu untersuchen, wie sich eine flächenhafte ambulante Palliativversorgung sowie die Sterbeorte von ambulanten Palliativpatienten in den Jahren nach Einführung der „Vereinbarung“ in WestfalenLippe entwickelten.

\section{Methodik}

Die Leistungsdaten der teilnehmenden Ärzte (Anzahl Patienten in Voll- und Teilversorgung sowie deren Betreuungsdauer und Sterbeorte) für 2012 bis 2014 wurden aus den quartalsweisen Meldungen der Leistungszahlen an die KVWL generiert. Die Daten der Jahre 2009/2010 (4. Quar- tal 2009 bis einschl. 3. Quartal 2010) und 2011 lagen bereits vor $[12,13]$ und wurden entsprechend integriert.

Um eine Entwicklung der Versorgungsstrukturen und deren Ergebnisse diskutieren zu können, erfolgte die Darstellung über 23 Regionen. Diese 23 Regionen umfassten 18 Kreise sowie 9 kreisfreie Städte, wobei 4 Kreise bzw. kreisfreie Städte über keinen eigenen PKD verfügten. In einzelnen dieser 23 Regionen organisierten sich PKD-Untereinheiten - meistens aufgrund der großen Fläche des Kreises und den damit verbundenen weiten Fahrtstrecken zum Patienten. Folglich konnten zur Zeit insgesamt 33 Einheiten getrennt betrachtet werden.

Im Rahmen der Weiterentwicklung der Strukturen kam es in den vergangenen Jahren zum Teil zu geringfügigen territorialen Änderungen bzw. zu geänderten ärztlichen Zuständigkeiten, so dass die Ergebnisse der einzelnen 23 bzw. 33 PKD untereinander nicht ohne weiteres über die Jahre verglichen werden können. Ferner ergaben sich auch durch die nunmehr synchrone Auswertung über fünf Jahre geringfügige Abweichungen zu den bereits publizierten Daten. Die Gesamtentwicklung im beobachteten $\mathrm{KV}$-Bereich ist hiervon jedoch nicht berührt.

Die jeweiligen Sterbeorte der Patienten wurden, ab dem Erhebungsjahr 2011, in einer der fünf Kategorien zugeordnet:

- Palliativstation

- Klinik (bzw. Normalstation)

- zu Hause

- Hospiz

- Alten- bzw. Pflegeheim

Ab 2014 wurde zusätzlich noch die Kategorie „Sonstiges“ eingeführt: Hierunter fallen Sterbeorte im Ausland oder Intensivbehandlungswohngemeinschaften. 2009/10 wurde Tod „zu Hause“ und „im Heim“ noch unter „zu Hause“ zusammengefasst. Ebenso wurde 2009/10 „Krankenhaus“ noch nicht in „Normalstation“ und
Tab. 1 Sterbeorte von Patienten, die 2009/10 bis 2014 im ambulanten Palliativnetzwerk WestfalenLippe betreut wurden.

\begin{tabular}{|llllll}
\hline Jahr & $\mathbf{2 0 0 9 / 1 0 *}$ & $\mathbf{2 0 1 1}$ & $\mathbf{2 0 1 2}$ & $\mathbf{2 0 1 3}$ & $\mathbf{2 0 1 4}$ \\
\hline gestorben & $\mathbf{3 5 3 7}$ & $\mathbf{6 4 1 5}$ & $\mathbf{9 2 8 3}$ & $\mathbf{1 1 2 8 9}$ & $\mathbf{1 3 8 8 5}$ \\
\hline Palliativstation & - & $137(2,1 \%)$ & $209(2,3 \%)$ & $292(2,6 \%)$ & $409(3,0 \%)$ \\
\hline Klinik & $470(13,4 \%)$ & $599(9,4 \%)$ & $818(8,9 \%)$ & $964(8,6 \%)$ & $1187(8,7 \%)$ \\
\hline zu Hause & $2480(70,5 \%)$ & $3505(55,0 \%)$ & $5336(58,3 \%)$ & $6100(54,5 \%)$ & $6130(44,7 \%)$ \\
\hline Hospiz & $566(16,1 \%)$ & $1192(18,7 \%)$ & $1329(14,5 \%)$ & $1323(11,8 \%)$ & $1731(12,6 \%)$ \\
\hline Heim & - & $942(14,8 \%)$ & $1457(15,9 \%)$ & $2504(22,4 \%)$ & $4256(31,0 \%)$ \\
\hline Sonstiges (\%) & - & - & - & - & $172(1,2 \%)$ \\
\hline Summe* & 3516 & 6375 & 9149 & 11183 & 13713
\end{tabular}

* IV/2009 bis III/2010, ** 2014 ohne „Sonstiges“, vorher noch nicht gesondert erfasst 
„Palliativstation“ differenziert. Aus diesem Grund wurden die Daten für 2009/10 nicht in die statistische Auswertung einbezogen.

Die Daten wurden in MS-Excel erfasst und ausgewertet. Um zu überprüfen, inwieweit Veränderungen der Anteile der einzelnen Sterbeorte im Zeitverlauf 2009-2014 statistisch signifikant sind, wurden Chi-Quadrat-Trend-Tests berechnet (SPSS Version 22). Dazu wurden die jährlichen Anteile der Sterbeorte „zu Hause“ und „im Heim“ zu „Häuslichkeit“ zusammengefasst und dann „Häuslichkeit“ vs. andere Sterbeorte über die Jahre 2009-2014 verglichen, ebenso „Klinik“ vs. andere.

\section{Ergebnisse}

Die Anzahl der Patienten, denen im Bereich der KVWL ein ambulantes Palliativ-Angebot gemacht wurde, stieg von 5682 in 2009/10 auf 17699 in 2014 kontinuierlich auf etwa das Dreifache an. Während 2009/10 noch 3537 Patienten innerhalb der Versorgungsstruktur starben, waren es 201413885 in 2014.

Bezogen auf die Sterbeorte zeigte sich ein Rückgang des Sterbeortes „Klinik“ (bezogen auf Normalstationen), hin zu den beiden „häuslichen“ Sterbeorten „zu Hause“ bzw. insbesondere „im Heim“ ( $\bullet$ Tab. 1). Aus Gründen der großen Datenmenge wird auf die Darstellung der Zahlen für die einzelnen PKD an dieser Stelle verzichtet und auf die Veröffentlichung auf der Seite des Berufsverbandes der Palliativmediziner in Westfalen-Lippe (www.bv-palliativmediziner.de) verwiesen.

Der gezeigte zeitliche Trend „Häuslichkeit“ vs. „andere" ist statistisch signifikant (Chi-Quadrat-Test „linear-mit-linear“, Wert der Prüfgröße 76,595, $\mathrm{df}=1$, zweiseitig, $\mathrm{p}<0,001$ ), wohingegen der Trend „Klinik“ vs. ,andere“ die Signifikanz knapp verfehlt (Chi-Quadrat-Test „linear-mit-linear“, Wert der Prüfgröße 3,012, df=1, zweiseitig, $p=0,083$ ).

In $>$ Abb. 1 ist die räumliche Verteilung der in 2014 der im Rahmen des im Palliativnetzwerks Westfalen-Lippe ambulant palliativmedizinisch versorgten bzw. verstorbenen Patienten dargestellt. Es zeigt sich eine Konzentration der betreuten Sterbefälle im westlichen Westfalen und eine homogenere Verteilung in den übrigen Landesteilen, mit Ausnahme des südöstlichen Randgebietes (wobei hier über Kreisgrenzen hinweg versorgt wird). Zu beachten ist, dass die jeweiligen Patientenzahlen den Adressen der jeweiligen Netzwerkstandorte zugeordnet wurden und sich die tatsächliche räumliche Abdeckung durch die teilnehmenden Arztpraxen breiter in der Fläche verteilt.

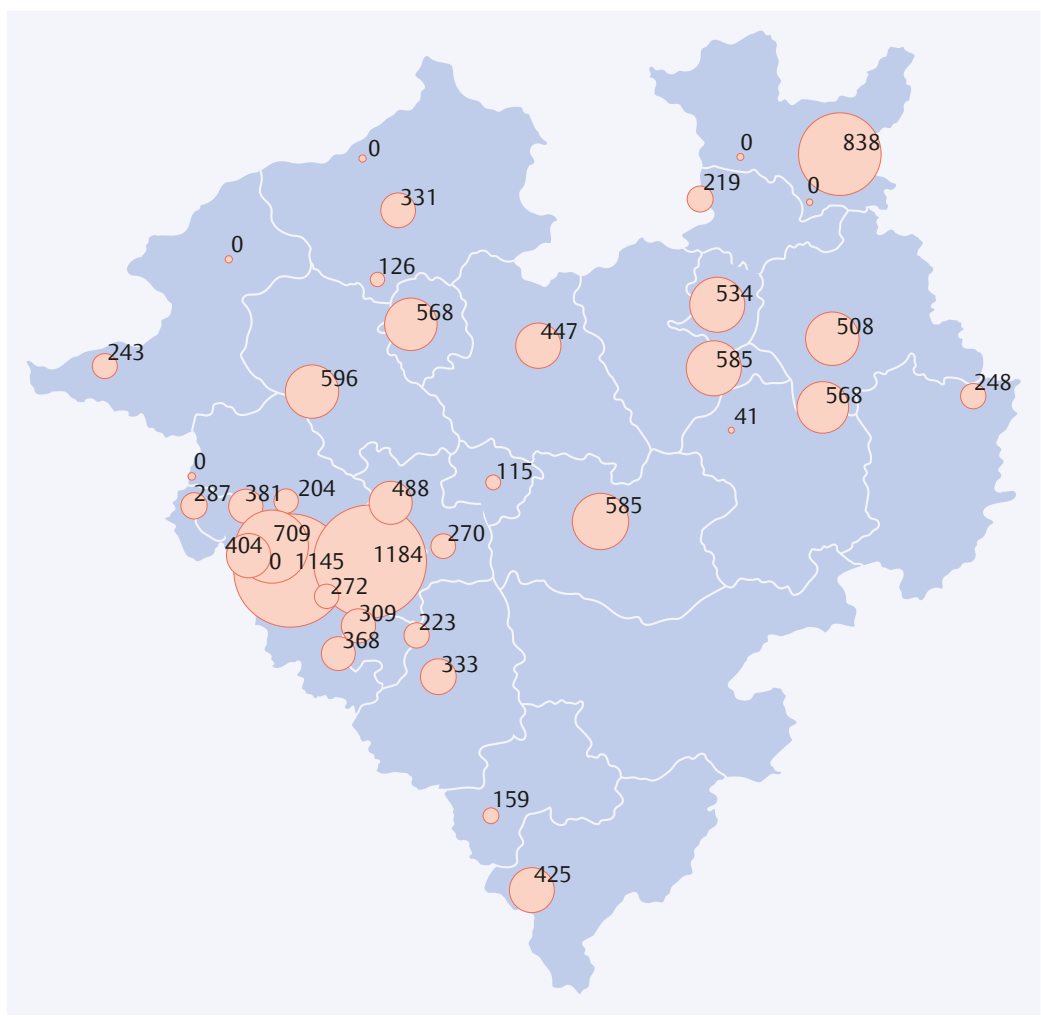

\section{Diskussion}

Viele Menschen wünschen sich, ihre letzte Lebensphase selbstbestimmt und in ihrer gewohnten Umgebung zu verbringen und hier auch zu sterben [1, 6]. Bereits aus Modellprojekten um das Jahr 2000 wurde ersichtlich, dass geeignete ambulante palliativmedizinische Strukturen diesem Wunsch mit gutem Ergebnis und mit überschaubarem finanziellem und personellem Aufwand entsprechen können. Hierbei lagen die Sterberaten zu Hause zwischen 60-64\% [5].

Diese Zahlen wurden im gesamten Bereich der KVWL überschritten - schon im Jahr 2010 starben hier um 70\%, 2014 bereits über 75\% der Patienten zu Hause, sofern sie in die palliativmedizinisch betreut wurden. Damit lag die häusliche Sterberate auch deutlich über den $61,7 \%$, die Lindena und Woskanjan im HOPE-Bericht 2011 beschreiben [11].

Aus der Region Halle/Saale berichtete ein SAPVTeam von einer häuslichen Sterberate von $91 \%$ bei 146 betreuten Patienten in einer Region mit ca. 500000 Einwohnern und einer Beobachtungszeit von einem Jahr [2]. Unsere Studie berichtet Daten der ambulanten palliativmedizinischen Versorgung aus Westfalen-Lippe mit 8,3 Millionen Einwohnern und jährlich etwa 91000 Sterbefällen.

- Wurden 2009/2010 6,2\% und 2011 8,2\% aller Sterbenden in Westfalen-Lippe durch ambulante palliativmedizinische Netzstrukturen betreut,
Abb. 1 Anzahl verstorbener Patienten, die $2014 \mathrm{im}$ Palliativnetzwerk WestfalenLippe ambulant betreut wurden (getrennt nach Regionen). 
- so waren es 2012 bereits $14,1 \%$,

- $201315,9 \%$ und

- 2014 19,4\%.

Es zeigt sich, dass mit der in Westfalen-Lippe gewählten Organisationsform eine hohe und weiter steigende Anzahl von Patienten ambulant palliativ versorgt werden kann.

Hohe Sterberaten zu Hause ließen sich in Westfalen-Lippe sowohl in dicht als auch in dünn besiedelten Gebieten beobachten, was zeigt, dass dieses System grundsätzlich geeignet ist, flächendeckend eingesetzt zu werden [12]. Qualitative Unterschiede sind jedoch wahrscheinlich dem noch immer unterschiedlichen Organisationsgrad und der Arbeitsweise der ambulanten palliativmedizinischen Strukturen geschuldet. Daher lohnt es sich, nach Homogenisierung der Versorgung den Zusammenhang zwischen Bevölkerungsdichte und Sterbeort erneut zu untersuchen. Bickel verweist auf eine Zunahme häuslicher Sterberaten in mehreren europäischen Ländern während der letzten zehn Jahre [1]. Allerdings spricht unsere Erfahrung dafür, dass Hausärzte in ländlichen Regionen eine festere Bindung zu ihren Patienten pflegen.

Trotz Stärkung ambulanter palliativer Versorgungsstrukturen dürfte das Sterben in Pflegeeinrichtungen zunehmen, Gründe dafür könnten sein:

- weiterer Ausbau von palliativmedizinischen Netzstrukturen

- demografischer Wandel

- Individualitätsmentalität der Sterbenden und ihrer Angehörigen

Dieser Entwicklung wird Rechnung getragen im 2014 veröffentlichten Eckpunktepapier des Gesundheitsministers [7] und dem Entwurf eines Gesetzes zur Verbesserung der Hospiz- und Palliativversorgung in Deutschland.

Die Anzahl der in Westfalen-Lippe in Pflegeeinrichtungen durch Palliativmediziner versorgter Patienten ist in den letzten Jahren stetig gestiegen. Dies ist jedoch nicht nur geänderten gesellschaftlicher Rahmenbedingungen, sondern auch der Tatsache geschuldet, dass Palliativmediziner mehr und mehr Patienten mit Nichttumorerkrankungen behandeln. Auch steigt die Akzeptanz hierfür sowohl seitens der Einrichtungen und Hausärzte als auch seitens der Patienten und der Angehörigen.

Die insgesamt beobachtete Zunahme der häuslichen Sterberate (eigene Wohnung und Heim) verlief naturgemäß in den betrachteten fünf Jahren nicht in allen Regionen gleich. Zwar kam es überwiegend zu Anstiegen, vereinzelt allerdings auch zu Abnahmen. Man könnte erwarten, dass mit der Zunahme der Zahl betreuter Patienten auch die Erfahrung der beteiligten Ärzte, Pflegenden und Koordinatoren zunimmt. Wir gehen jedoch davon aus, dass die qualitative Ausgestal- tung der praktischen Tätigkeit in den einzelnen Regionen noch immer sehr unterschiedlich ist. So war in einer Region eine starke Schwankung in der Anzahl betreuter Patienten zwischen 2010 und 2012 zu sehen. Grund war eine personelle Neuorganisation dieses PKD.

Ein weiterer möglicher Grund könnte auch die Anzahl der Koordinatoren sein, die nicht proportional mit der Fallzahl angewachsen ist: Gemäß der „Vereinbarung“ müssen

- grundsätzlich mindestens eine,

- bei mehr als 75 Patienten pro Quartal mindestens anderthalb und

- ab über 125 Patienten/Quartal mindestens zwei

Koordinatoren-Stellen mit 3-jährig ausgebildeten, examinierten Pflegefachkräften besetzt sein [9]. Steigt die Anzahl der betreuten Patienten weiter, bleibt die Anzahl der Koordinatoren konstant. Einzelne PKD mit hohen Patientenzahlen beschäftigen auch mehr Koordinatoren als vorgeschrieben (persönliche Mitteilung).

Die regionalen Unterschiede hinsichtlich des Vorhandenseins von Palliativstationen oder stationären Hospizen haben einen deutlichen Einfluss auf den Sterbeort: In zwei Regionen waren stationäre Hospize nicht verfügbar, entsprechend lagen hier die Hospiz-Sterberaten mit 0,8 bzw. 2,2\% verhältnismäßig niedrig. Durch die Einrichtung eines stationären Hospizes und der vermehrten Nutzung dieses Angebots sank die Zahl der im häuslichen Umfeld sterbenden Patienten in einer Region. In einer anderen Region hatte ein stationäres Hospiz eine sehr hohe Akzeptanz. Die Anzahl der Patienten, die auf Palliativstationen starben, war gering, erhöhte sich jedoch mit der Zunahme von Palliativbetten in den Kliniken. Die Qualität der Palliativversorgung bemisst sich schließlich nicht nur danach, inwieweit Patienten an ihrem Wunschort sterben, sondern daran, ob jedem Patienten auch ein bedarfsgerechtes palliativmedizinisches Angebot zur Verfügung steht. Dazu gehört, dass der Leistungsanspruch auf SAPV nach $\S$ 37b SGB V im Bedarfsfall unverzüglich erfüllt wird.

2014 wurden 19,4\% Menschen in Westfalen-Lippe in ihrer letzten Lebensphase gemäß der Vereinbarung betreut. Waren im Jahr 2010 noch 9 Palliativnetze nicht in der Lage, Daten für eine strukturierte Auswertung zu liefern [12], waren dies im Jahr 2011 nur noch 3 [13]. Seit 2012 kann festgestellt werden, dass in allen politischen Kreisen und kreisfreien Städten in Westfalen-Lippe ambulante palliativmedizinische Strukturen etabliert sind - wenngleich auf unterschiedlichem Entwicklungsstand. In 22 von 23 PKD konnte die Zahl der betreuten Patienten zwischen 2009/2010 und 2014 gesteigert werden, lediglich in einer Region konnte sich ein PKD nicht stabil entwickeln und ging in den Nachbarregionen auf. 
Nicht selten änderten sich auch zwischen einzelnen PKD die Versorgungsgebiete - bedingt z.B. durch Wohnorte der PKD-Ärzte oder geografische Gegebenheiten.

Es wurden in Westfalen-Lippe aber 50\% mehr Patienten ein Kontakt zu einem Palliativmediziner ermöglicht, als dies mit 10\% der Patienten am Lebensende in den Empfehlungen des GKV-Spitzenverbandes nach $\S 132$ Abs. 2 SGB V für die spezialisierte ambulante Palliativversorgung für notwendig beschrieben wurde [4].

Der Abschluss von Verträgen zwischen Krankenkassen und den Leistungserbringern - auch wenn er inzwischen in Deutschland in vielen Bereichen geschieht - gewährleistet für sich noch keine bedarfsgerechte palliativmedizinische Versorgung für den einzelnen Patienten. Die geschaffenen Strukturen müssen es ermöglichen, dem anspruchsberechtigten Patienten unmittelbar und niederschwellig Zugang zu der seinem Bedarf entsprechenden Versorgungsform zu ermöglichen. Im Bereich der KVWL ist dieses wichtige Qualitätskriterium erfüllt.

In Westfalen-Lippe konnte die Anzahl der Patienten in der ambulanten Palliativversorgung innerhalb von 4 Jahren verdreifacht werden. Dies unterstreicht die hohe Akzeptanz der Versorgung durch ambulant tätige Haus- und Fachärzte, so wie dies bereits Schneider et al. [14] beschrieben. Damals äußerten $86 \%$ der Hausärzte den Wunsch, auch bei Patienten in der letzten Lebensphase aktiv in der Patientenversorgung integriert zu bleiben. Sie hielten die komplette Abgabe der Patientenbetreuung an ein SAPV-Team nicht für wünschenswert.

Nach dem richtungsweisenden Artikel von Temel et al. im Jahr 2010 wurde eine frühe Kooperation von Palliativmedizinern und Onkologen gefordert [15]. Dies beschreiben auch Quill und Abernethy [16] und fordern, gerade im ambulanten Bereich durch niederschwellige Möglichkeiten der Kooperation zwischen Haus- und Fachärzten die ambulante palliative Patientenversorgung zu stärken. Mit der Einführung palliativmedizinischer Leistungsziffern in den neuen Katalog des Einheitlichen Bewertungsmaßstabes wird diese Entwicklung unterstrichen.

Im gesamten Bereich der KVWL können seit fünf Jahren auch Patienten ohne ausgeprägte Symptomatik ambulant palliativmedizinisch versorgt werden. Nach der Aufbauphase wurde ein flächendeckendes Versorgungsangebot installiert, allerdings schwanken die häuslichen Sterberaten zwischen den einzelnen PKD-Regionen noch. Im Jahr 2013 wurden 15,9\%, im Jahr 2014 19,4\% aller Verstorbenen ambulant palliativmedizinisch betreut, womit ein Beitrag zu Umsetzung der Charta zur Betreuung Sterbender geleistet wurde [3].
Das westfälisch-lippische Modell basiert auf der Annahme, dass der Hausarzt für seine Patienten auch dann zentraler Ansprechpartner bleibt, wenn diese in eine palliative Situation kommen. Als besonders wichtige Voraussetzung wird das oft über Jahre bis Jahrzehnte gewachsene Vertrauensverhältnis zwischen Patient und Hausarzt gesehen, das gerade in der letzten Lebensphase nicht ohne Grund unterbrochen werden soll [8]. Wichtigste Säule des Modells ist daher die Teilnahme von rund $90 \%$ aller in Westfalen-Lippe niedergelassener Hausärzte, unterstützt durch 33 palliativmedizinische Konsiliardienste mit ca. 260 Palliativmedizinern und über 100 koordinierenden Palliativpflegefachkräften. Sie alle schaffen auf diese Weise ein niedrigschwelliges, effizientes und rund um die Uhr verfügbares Angebot für ihre Patienten, das gleichzeitig auf regionale Besonderheiten und bereits vorhandene Strukturen Rücksicht nimmt.

Berücksichtigt man alle Akteure, so besteht dieses Netzwerk aus qualifizierten Palliativärzten Palliativpflegekräften, Mitarbeitern der ambulanten Hospize und anderer Professionen wie Physiotherapeuten, Psychoonkologen und Seelsorger. Durch die Verantwortung in denselben Händen werden Schnittstellenprobleme und Unsicherheiten der Zuständigkeit in verschiedenen Krankheitsphasen vermieden.

\section{Konsequenz für Klinik und Praxis}

- Eine jederzeit erreichbare ambulante palliativmedizinische Versorgung ermöglicht es vielen Patienten, ihrem Wunsch entsprechend, zu Hause oder im Pflegeheim zu sterben.

- Fünf Jahre nach Einführung strukturierter AAPV und SAPV wurde bereits jeder 5. Patient am Lebensende ambulant palliativmedizinisch betreut.

- Es bestehen regionale Unterschiede in der Versorgungsdichte und -qualität, die durch konsequente Angebotserweiterung und Qualitätssicherung ausgeglichen werden können.

\section{Literatur}

1 Bickel H. Das letzte Lebensjahr: Eine Repräsentativstudie an Verstorbenen - Wohnsituation, Sterbeort und Nutzung von Versorgungsangeboten. Z Gerontol Geriat 1998; 31: 193-204

2 Bretschneider K, Kasprick L, Luderer C. "Elisabeth Mobil mbH” - die spezialisierte ambulante Palliativversorgung im Raum Halle (Saale) - eine wissenschaftliche Auswertung. Z Palliativmed 2012; 13: 36-46

3 Bundesärztekammer, Deutscher Hospiz- und PalliativVerband e. V., Deutsche Gesellschaft für Palliativmedizin e. V. Charta zur Betreuung Sterbender http://www.charta-zur-betreuung-sterbender.de Letzter Zugriff am 19.02.2016 
4 GKV-Spitzenverband. Empfehlungen des GKVSpitzenverbandes nach § 132 d Abs. 2 SGB V für die spezialisierte ambulante Palliativversorgung vom 23. 06. 2008 in der Fassung vom 05. 11. 2012 http://www.gkv-spitzenverband.de/media/ dokumente/krankenversicherung_1/hospiz_palliativversorgung/Palliativ_Empfehlungen nach_132d_Abs_2 SGB_V_05-11-20102.pdf Letzter Zugriff am 19.02.2016

5 Deutscher Bundestag. Enquete-Kommission Ethik und Recht der modernen Medizin: Verbesserung der Versorgung Schwerstkranker und Sterbender in Deutschland durch Palliativmedizin und Hospizarbeit, Drucksache 15/5858, 22.06.2005

http://dipbt.bundestag.de/doc/ btd/15/058/1505858.pdf

Letzter Zugriff am 19.02.2016

6 Gomes B, Calanzani N, Higginson II. Reversal of the British trends in place of death: time series analysis 2004-2010. Palliat Med 2012; 26: 102-107

7 Gröhe H, Widmann-Mauz A, Spahn J et al. Verbesserung der Hospiz- und Palliativversorgung in Deutschland. Eckpunktepapier http://www.bmg.bund.de/ministerium/meldungen/2015/hospiz-und-palliativversorgung-indeutschland.html

Letzter Zugriff am 19.02.2016

8 Hoberg C, Junker D, Lux EA et al. Befragung von Zugehörigen verstorbener Palliativpatienten in der ambulanten Versorgung des Palliativnetzes Bielefeld e. V. Munch Med Wochenschr 2015; 157 (Suppl 5): 25-31

9 Kassenärztliche Vereinigung Westfalen-Lippe. Vereinbarung zur Umsetzung der ambulanten palliativmedizinischen Versorgung von unheilbar erkrankten Patienten im häuslichen Umfeld http://www.kvwl.de/arzt/recht/kvwl/palliativ/ palliativ_vereinbarung.pdf Letzter Zugriff am 19.02.2016
10 Landesbetrieb Information und Technik NordrheinWestfalen. Gestorbene in Nordrhein-Westfalen 2005 bis 2013

https://www.it.nrw.de/presse/pressemitteilungen/2014/pdf/256_14.pdf Letzter Zugriff am 19.02.2016

11 Lindena G, Woskanjan S. Hospiz- und PalliativErfassung Bericht. HOPE 2011 https://www.hope-clara.de/download/Bericht_ HOPE_2011.pdf Letzter Zugriff am 03.03.2016

12 Lux EA, Althaus A, Claßen B et al. Ambulante Palliativversorgung in Westfalen-Lippe - Strukturierte Bestandsaufnahme 12 Monate nach Inkrafttreten der „Vereinbarung zur Umsetzung der ambulanten palliativmedizinischen Versorgung von unheilbar Kranken im häuslichen Umfeld“. Z Palliativmed 2012; 13: 97-102

13 Lux EA, Althaus A Claßen B et al. Ambulante Palliativversorgung in Westfalen-Lippe - strukturierte Bestandsaufnahme 12 und 36 Monate nach Inkrafttreten der „Vereinbarung zur Umsetzung der ambulanten palliativmedizinischen Versorgung von unheilbar Kranken im häuslichen Umfeld“. Munch Med Wochenschr 2013; 155 (Suppl. 4): 44-50

14 Schneider N, Engeser P, Behrmann M et al. Spezialisierte ambulante Palliativversorgung - Die Erwartung der Hausärzte. Schmerz 2011; 25: $168-173$

15 Temel JS, Greer JA, Muzikansky A et al. Early palliative care for patients with metastatic non-small-cell lung cancer. N Engl J Med 2010; 363 : 733-742

16 Quill TE, Abernethy AP. Generalist plus specialist palliative care - creating a more sustainable model. N Engl J Med 2013; 368: 1173-1175
Interessenkonflikt

Die Autoren geben an, dass kein Interessenkonflikt besteht.

DOI 10.1055/s-0041-106677 Dtsch Med Wochenschr 2016; 141: e67-e72

(c) Georg Thieme Verlag KG . Stuttgart · New York .

ISSN 0012-0472 